\title{
The relationship between J wave and ventricular tachycardia during Takotsubo cardiomyopathy
}

Seong Huan Choi ${ }^{\dagger}$, Oh-Hyun Lee ${ }^{\dagger}$, Gwang-Seok Yoon, Sung Woo Kwon, Sung-Hee Shin, Sang-Don Park, Seong-III Woo, Jun Kwan, Dae-Hyeok Kim ${ }^{*+}$ and Yong-Soo Baek ${ }^{*+}$ (])

\begin{abstract}
Background and objectives: Takotsubo cardiomyopathy (TTC) occasionally causes life-threatening ventricular arrhythmia. J wave on surface electrocardiography (sECG) has also been associated with idiopathic ventricular fibrillation and cardiac events; therefore, we investigated whether the presence of J wave on sECG is a potential risk factor for ventricular arrhythmia in patients with TTC.
\end{abstract}

Subjects and methods: We performed a retrospective study in 79 patients who were diagnosed with TTC from 2010 to 2014. Among them, 20 (25.3\%) were diagnosed with ventricular tachycardia (VT). The J wave on the sECG was defined as I point elevation manifested through QRS notching or slurring at least $1 \mathrm{~mm}$ above the baseline in at least two leads.

Results: A higher prevalence of ventricular tachycardia was observed in patients with J wave. The corrected QT interval (QTC) was significantly longer in the VT group than in the non-VT group. In a multivariate analysis, the presence of J wave appeared to be the only independent predictors of VT [Hazard Ratio (HR) 3.5, $p=0.019$ ].

Conclusion: Our results suggest that the presence of $\mathrm{J}$ wave on the sECG is significantly associated with VT, and appear to indicate that the presence of $\mathrm{J}$ wave is a strong and independent predictor of VT in patients with TTC.

Keywords: J wave, Ventricular tachycardia, Takotsubo cardiomyopathy

\section{Introduction}

Takotsubo cardiomyopathy (TTC) or apical ballooning syndrome is a form of reversible cardiomyopathy mimicking the symptomatology and electrocardiographic findings of acute myocardial infarction (AMI) without significant coronary artery disease on angiography [1]. Furthermore, life-threatening ventricular tachyarrhythmias

\footnotetext{
*Correspondence: kdhmd@inha.ac.kr; existsoo@inha.ac.kr

t'seong Huan Choi and Oh-Hyun Lee have contributed equally to this work and are joint first authors.

${ }^{\dagger}$ Dae-Hyeok Kim and Yong-Soo Baek have contributed equally to this work.

Division of Cardiology, Department of Internal Medicine, Inha University College of Medicine and Inha University Hospital, 27 Inhang-ro, Jung-gu, Incheon 22332, Republic of Korea
}

caused by repolarization abnormalities and QTc prolongation, including torsades de pointes (TdP), may be seen in up to $8 \%$ of TTC patients [2]. The J point and ST-segment elevation which sometimes manifests as a notch or slur of the QRS (J wave) is the characteristic ECG pattern of early repolarization (ER). Moreover, recent studies have demonstrated that J wave on the SECG is associated with ventricular tachycardia (VT) and fibrillation (VF) in an experimental model consisting of canine ventricular wedge preparations [3]. This concept has now expanded to include other structural heart diseases such as AMI, variant angina, and even some forms of cardiomyopathy [4]. In spite of the increasing importance of J wave, its significance in TTC patients with ventricular arrhythmia is 
unknown. In this study, we aimed to investigate whether the presence of J wave on the SECG is a potential risk factor for ventricular arrhythmia in patients with TTC.

\section{Subjects and methods \\ Patient selection and data collection}

We performed a retrospective single-center study which included 79 patients (mean age $=66.4 \pm 15.3$ years, 38 males) who were diagnosed with TTC from 2010 to 2014. We collected data from consecutive patients who were classified into two groups based on the presence or absence of ventricular tachycardia (VT group: $n=20$ [25.3\%], non-VT group: $n=59$ [74.6\%]). VT group patients were admitted to intensive care unit (ICU); therefore, continuous ECG monitoring was used to detect any arrhythmia. The diagnosis of TTC was made according to the Mayo Clinic criteria [5]. Clinical and echocardiographic parameters; precipitating factors and subtypes of TTC; peak cardiac enzyme and electrolyte levels were examined. The inclusion criteria for TTC in our study were (1) transthoracic echocardiogram consistent with ABS/TTC (transient hypokinesis, akinesis or dyskinesis of the left ventricular mid-segments with or without apical involvement; the regional wall motion abnormality extend beyond a single epicardial vascular distribution), and (2) new ECG abnormality (either STsegment elevation and/or T-wave inversion) or modest elevation in cardiac troponin. Exclusion criteria were (1) transechocardiogram consistent with ischemic cardiomyopathy or dilated cardiomyopathy and (2) presences of obstructive coronary disease or angiographic evidence of acute coronary syndrome [5]. This study was approved by the Institutional Review Board at Inha University Hospital, which waived the requirement of obtaining written informed consent from patients.

\section{ECG analysis}

All ECGs obtained during hospitalization were reviewed and analyzed by three independent investigators, who had expertise in cardiology, to confirm the correct J wave pattern. Presence of J wave was confirmed only if the three reviewer's observations were coherent. Twelve-lead ECGs were downloaded from the GE Marquette MUSE system (GE Medical Systems, Milwaukee, WI, USA) and analyzed digitally (Adobe Acrobat X professional; Adobe systems Incorporated, San Jose, CA, USA). J wave was defined as an elevation of QRS-ST junction (J point) in at least two leads on a resting 12-lead ECG, as described previously [6]. The amplitude of J point elevation had to be at least $1 \mathrm{~mm}(0.1 \mathrm{mV})$ above the isoelectric line, either as QRS slurring (smooth transition from the QRS segment to the ST segment), or notching (positive J deflection inscribed on the $S$ wave) in at least two consecutive inferior leads (II, III, and aVF), lateral leads (I, aVL, and V4-V6), or both. We included patients with Brugada pattern showing J wave in the anterior precordial leads (V1V3) on the ECG, and measured the amplitude of J wave at the peak of the positive deflection, and at the QRS-ST junction in cases in which notched and slurred ECG pattern was observed, respectively.

\section{Statistical analysis}

Statistical analyses were performed using the SPSS statistical software (SPSS version 21.0 for Windows, SPSS Inc., Chicago, IL, USA). Continuous variables were expressed as mean $\pm \mathrm{SD}$ and compared using the Student $t$ test or Mann-Whitney $U$ test, as appropriate. Categorical variables were expressed as percentages and compared using Chi-square or Fisher's exact tests, as appropriate. Univariate and multivariate Cox regression analyses were performed to identify a subset of predictor variables for VT. Results showing a $p$ value $<0.05$ (2-tailed) were considered statistically significant.

\section{Results \\ Baseline characteristics}

Table 1 shows the baseline characteristics of the study population. Among the 20 patients in the VT group, 13 and 7 were male and female, respectively. The mean age in the VT group was $69 \pm 13.8$ years. The non-VT group consisted of 59 patients and included 24 males and 35 females. The mean age of the non-VT group was $66 \pm 15.8$ years and was not statistically different from that of the VT group $(p=0.55)$.

With the exception of cardiogenic shock, two groups did not statistically differ in terms of clinical presentation. Cardiogenic shock was significantly more common in the VT group $(n=9,45 \%)$ than in the non-VT group $(n=12,20 \%)(p=0.028)$. VT group had higher mortality rate compared to non-VT group ( $40.2 \%$ vs. $13.3 \%$, HR $3.7,95 \% \mathrm{CI}, 1.4 \pm 9.9, p=0.009)$. No statistical differences were observed between the two groups in terms of risk factors for cardiac disease, including diabetes mellitus, hypertension, smoking, and low-density lipoprotein cholesterol (LDL-C) level; or in terms of precipitating factors of TTC, including emotional triggers and physical triggers such as asthma attack, ischemic stroke, bleeding, sepsis, surgical procedures, malignancy, and acute kidney injury [7].

\section{Laboratory, ECG, and echocardiographic findings}

Table 2 shows ECG and laboratory findings in the study population. The QTc interval was significantly longer in the VT group ( $577.0 \pm 58.5 \mathrm{~ms})$ than in the non-VT group $(534.2 \pm 61.1 \mathrm{~ms})(p=0.01)$. No statistical difference in electrolyte levels; cardiac enzymes; and prevalence of atrial 
Table 1 Baseline characteristics of patients in the VT group and Non-VT group

\begin{tabular}{|c|c|c|c|c|}
\hline & Total $(n=79)$ & VT group $(n=20)$ & Non-VT group $(n=59)$ & $P$ value \\
\hline Age, years & $66.4 \pm 15.3$ & $68.6 \pm 13.8$ & $65.6 \pm 15.7$ & 0.55 \\
\hline Male gender, $n(\%)$ & $37(47)$ & $13(65)$ & $24(41)$ & 0.07 \\
\hline Body surface area, $\mathrm{m}^{2}$ & $1.5 \pm 0.1$ & $1.6 \pm 0.1$ & $1.5 \pm 0.1$ & 0.58 \\
\hline \multicolumn{5}{|l|}{ Cardiac risk factor } \\
\hline Diabetes, $n(\%)$ & $19(25)$ & $5(25)$ & $14(25)$ & 1.00 \\
\hline Hypertension, n (\%) & $40(50)$ & $8(40)$ & $32(53)$ & 0.30 \\
\hline Smoker, n (\%) & $20(25)$ & $5(25)$ & $15(25)$ & 1.00 \\
\hline LDL-C, mg/dL & $83.2 \pm 41.9$ & $86.0 \pm 43.7$ & $75.7 \pm 37.8$ & 0.42 \\
\hline \multicolumn{5}{|l|}{ Trigger } \\
\hline \multicolumn{5}{|l|}{ Physical triggers } \\
\hline Asthma attack, n (\%) & $3(4)$ & $1(5)$ & $2(3)$ & 0.73 \\
\hline Ischemic stroke, n (\%) & $3(4)$ & $0(0)$ & $3(5)$ & 0.31 \\
\hline Bleeding, $n(\%)$ & $4(5)$ & $2(10)$ & $2(3)$ & 0.24 \\
\hline Sepsis, $n(\%)$ & $50(64)$ & $13(65)$ & $37(63)$ & 0.89 \\
\hline Surgical procedures, $n \%$ ) & $3(4)$ & $2(10)$ & $1(2)$ & 0.09 \\
\hline Malignancy, n (\%) & $2(3)$ & $1(5)$ & $1(2)$ & 0.41 \\
\hline AKI, $n(\%)$ & $6(8)$ & $0(0)$ & $6(10)$ & 0.14 \\
\hline \multicolumn{5}{|l|}{ Emotional triggers } \\
\hline Depression, $n(\%)$ & $1(1)$ & $0(0)$ & $1(2)$ & 0.56 \\
\hline Anxiety, $n(\%)$ & $2(3)$ & $0(0)$ & $2(3)$ & 0.41 \\
\hline Suicide attempt, $n$ (\%) & $1(1)$ & $1(5)$ & $0(0)$ & 0.08 \\
\hline Idiopathic, n (\%) & $4(5)$ & $0(0)$ & $4(7)$ & 0.24 \\
\hline \multicolumn{5}{|l|}{ Clinical presentation } \\
\hline Chest pain, $n(\%)$ & $22(27)$ & $4(20)$ & $18(30)$ & 0.76 \\
\hline Dyspnea, $n(\%)$ & $34(42)$ & $6(30)$ & $28(46)$ & 0.57 \\
\hline Nausea/vomiting, n (\%) & $4(5)$ & $1(5)$ & $3(5)$ & 1.00 \\
\hline Palpitations, $n(\%)$ & $5(6)$ & $2(10)$ & $3(5)$ & 0.27 \\
\hline Loss of consciousness, $n$ (\%) & $5(6)$ & $4(20)$ & $1(1)$ & 0.06 \\
\hline Cardiogenic shock, n (\%) & $21(26)$ & $9(45)$ & $12(20)$ & 0.028 \\
\hline Pulmonary edema, $n$ (\%) & $52(65)$ & $15(75)$ & $37(61)$ & 0.59 \\
\hline
\end{tabular}

Values are mean \pm SD or number (\%); VT ventricular tachycardia; CRF chronic renal failure; $L D L$ - $C$ low-density lipoprotein cholesterol; others idiopathic; $P$ value by Student $t$-Test and Mann-Whitney $U$ test

arrhythmia, bradycardia, and other electrocardiographic changes; including ST-segment elevation, $T$ wave inversion, and $q$ wave, were observed between two groups. Similarly, echocardiographic findings and phenotypes between the VT and non-VT group were not significantly different between the groups (Table 3).

Patients with J wave had a significantly higher prevalence of ventricular tachycardia ( $53 \%$ vs. $8 \%, p<0.001)$ and polymorphic VT or $\operatorname{TdP}(n=11,36 \%)$ than those without J wave $(n=3,6 \%)(p=0.001)$. However, the prevalence of monomorphic VT did not statistically differ between the groups $(p=0.19)$.

\section{Detailed characteristics of the $J$ wave for the prediction of VT}

Figure 1 shows baseline ECGs of patients with TTC. Figures 1a, 2a show a notched J wave (arrows) in inferior leads (II, III, aVF) in patients who had an event of monomorphic (Fig. 1b) or polymorphic VT (Fig. 2b). Table 4 shows that the prevalence of J wave was significantly higher in the VT group $(n=15,75 \%)$ than in the non-VT group $(n=14,25 \%)(p=0.001)$. Moreover, the prevalence of $J$ wave with a notched pattern was significantly higher in the VT group than in the non-VT $(n=13,65 \%$ vs. $n=13,21 \% ; p=0.001)$. In addition, a J 
Table 2 ECG and laboratory findings in the VT and Non-VT group

\begin{tabular}{|c|c|c|c|c|}
\hline & Total $(n=79)$ & VT group $(n=20)$ & Non-VT group $(n=59)$ & $P$ value \\
\hline \multicolumn{5}{|l|}{ Electrocardiographic changes } \\
\hline Atrial arrhythmia, $n(\%)$ & $26(33)$ & $12(60)$ & $14(25)$ & 0.003 \\
\hline AV block, $n(\%)$ & $2(2)$ & $0(0)$ & $2(3)$ & 1.000 \\
\hline ST-segment elevation, $n(\%)$ & $36(45)$ & $9(45)$ & $27(45)$ & 1.000 \\
\hline T-wave inversion, $n(\%)$ & $55(68)$ & $16(80)$ & $39(65)$ & 0.27 \\
\hline Q wave, $n(\%)$ & $18(22)$ & $4(20)$ & $14(23)$ & 0.51 \\
\hline QTc interval, ms & $545.2 \pm 63.6$ & $577.0 \pm 58.5$ & $534.2 \pm 61.1$ & 0.01 \\
\hline \multicolumn{5}{|l|}{ Laboratory findings } \\
\hline Sodium, mmol/L & $139.1 \pm 4.9$ & $139.6 \pm 5.9$ & $138.9 \pm 4.6$ & 0.59 \\
\hline Potassium, mmol/L & $4.1 \pm 0.7$ & $3.9 \pm 0.8$ & $4.1 \pm 0.7$ & 0.64 \\
\hline lonized calcium, mg/dL & $0.96 \pm 0.1$ & $0.95 \pm 0.1$ & $0.97 \pm 0.1$ & 0.51 \\
\hline Magnesium, mg/dL & $2.1 \pm 0.4$ & $2.1 \pm 0.4$ & $2.2 \pm 0.4$ & 0.16 \\
\hline \multicolumn{5}{|l|}{ Cardiac enzymes } \\
\hline Peak CK, ng/mL & $827.2 \pm 1409.9$ & $1008.5 \pm 1634.4$ & $766.7 \pm 1336.7$ & 0.51 \\
\hline Peak CK-MB, ng/mL & $30.7 \pm 46.9$ & $49.1 \pm 69.7$ & $24.7 \pm 35.1$ & 0.15 \\
\hline Peak troponin-l, ng/mL & $9.8 \pm 20.6$ & $16.5 \pm 34.1$ & $7.7 \pm 13.3$ & 0.28 \\
\hline NT-proBNP, pg/mL & $12900.2 \pm 13370.2$ & $14179.8 \pm 13207.2$ & $12293.6 \pm 13499.0$ & 0.28 \\
\hline
\end{tabular}

Values are mean \pm SD or number (\%); VT ventricular tachycardia; Atrial arrhythmia: PSVT, atrial tachycardia, atrial fibrillation; $C K$ creatine kinase; $C K-M B$ creatine kinasemyocardial band; NT-proBNP N-terminal pro-brain natriuretic peptide

Table 3 TTE findings in the VT group and Non-VT group

\begin{tabular}{lllll}
\hline & Total $(\boldsymbol{n = 7 9 )}$ & VT group $(\boldsymbol{n}=\mathbf{2 0})$ & Non-VT group $(\boldsymbol{n}=\mathbf{5 9 )}$ & $\boldsymbol{P}$ value \\
\hline Phenotype & & & & \\
Classic TTC pattern, $n(\%)$ & $45(56)$ & $12(60)$ & $33(55)$ & $7(13)$ \\
Apical sparing TTC pattern, $n(\%)$ & $12(16)$ & $5(25)$ & $0(0)$ & 0.81 \\
Isolated basal LV dysfunction, $n(\%)$ & $0(0)$ & $0(0)$ & $8(13)$ & 0.29 \\
Global LV hypokinesis & $8(10)$ & $0(0)$ & $11(18)$ & 0.19 \\
Others & $14(17)$ & $3(15)$ & $34.8 \pm 6.6$ \\
Echocardiographic parameters & & & $49.5 \pm 5.1$ \\
LVEF (\%) & $35.0 \pm 7.6$ & $35.7 \pm 10.3$ & $38.3 \pm 7.5$ \\
LVEDD (mm) & $48.9 \pm 5.4$ & $47.1 \pm 6.4$ & $41.9 \pm 7.5$ \\
LVESD (mm) & $37.6 \pm 7.4$ & $35.4 \pm 6.9$ & $14.6 \pm 8.1$ \\
LAD (mm) & $41.7 \pm 7.3$ & $41.1 \pm 6.9$ & 0.73 \\
E/E' & $14.2 \pm 7.5$ & $12.3 \pm 3.3$ & 0.13 \\
\hline
\end{tabular}

Values are mean \pm SD or number (\%); TTE transthoracic echocardiography; VT ventricular tachycardia; TTC Takotsubo cardiomyopathy; LVEF left ventricular ejection fraction; LVEDD left ventricular end-diastolic diameter; LVESD left ventricular end-systolic diameter; LAD left atrial diameter; E/E' early diastolic mitral inflow velocity/ early diastolic tissue Doppler velocity

wave in the inferior leads or a J point elevation $\geq 0.2 \mathrm{mV}$ were significantly more common in the VT group $(n=9,45 \%$ vs. $n=12,20 \% ; p=0.04, n=9,45 \%$ vs. $n=2,3 \% ; p=0.001$, respectively). Similarly, J wave in the inferolateral leads with a point elevation $\geq 0.2 \mathrm{mV}$ was also significantly higher in the VT group $(n=8$, $40 \%$ vs. $n=2,3 \% ; p<0.001)$. Likewise, the prevalence of horizontal/descending ST segment after the J point was significantly higher in the VT group ( $n=13,65 \%$ vs. $n=11,18 \%)$. However, no significant difference in the prevalence of ascending ST segment after the J wave was observed between the two groups.

Univariate Cox regression analysis demonstrated that QTc duration and presence of J wave were significantly associated with the occurrence of VT (HR 1.0; $p=0.002$, HR 5.2; $p=0.002$, respectively). Multivariate Cox regression analysis showed that the presence of $\mathrm{J}$ 


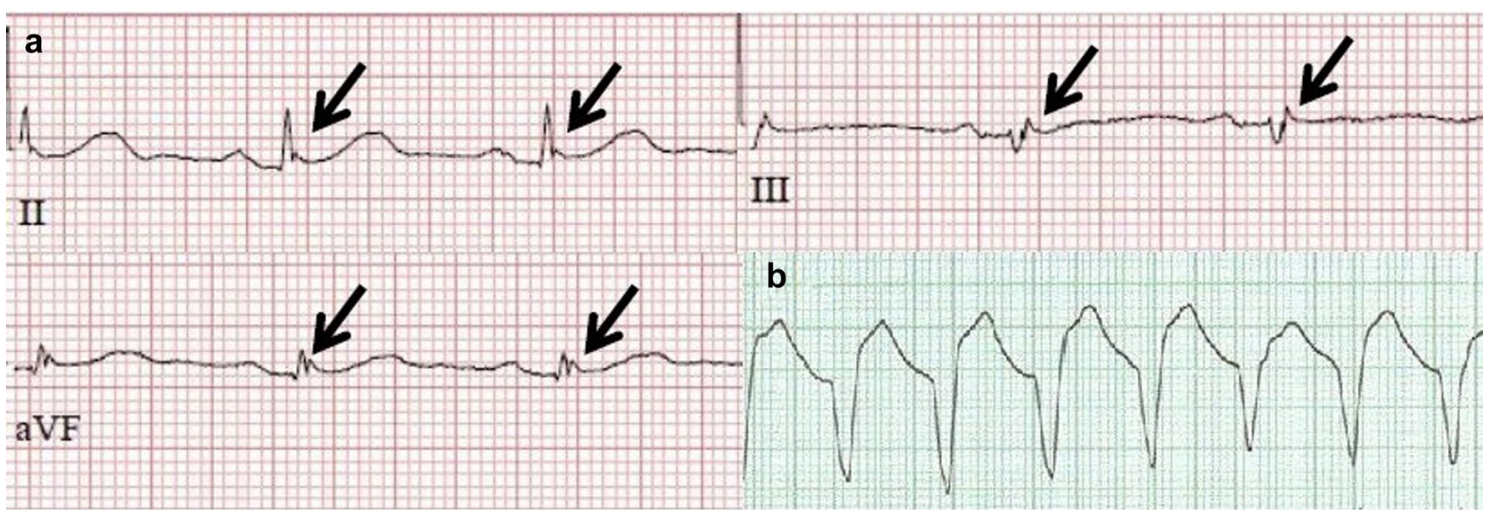

Fig. 1 Baseline ECGs in TTC patients. (a) Notched elevation (arrows) in inferior leads (II, III, aVF) in patients who experienced an event of monomorphic ventricular tachycardia (b)

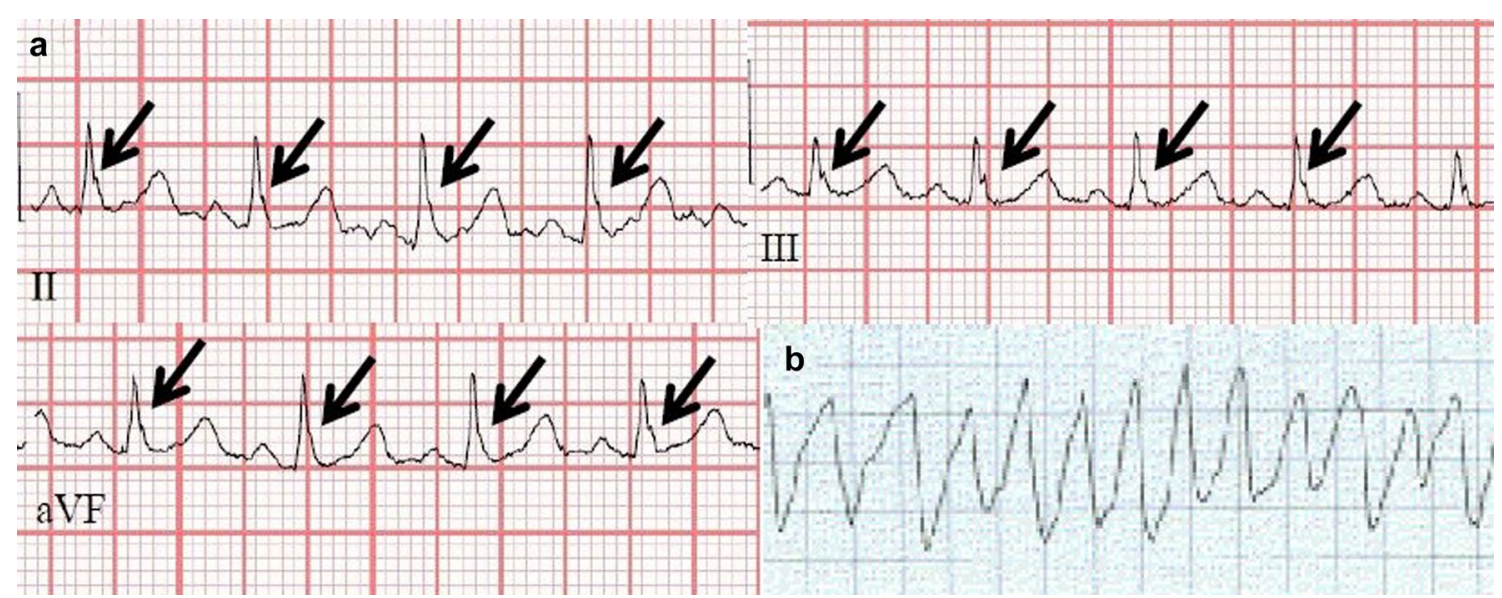

Fig. 2 Baseline ECGs in TTC patients. (a) Notched elevation (arrows) in inferior leads (II, III, aVF) in patients who experienced an event of polymorphic ventricular tachycardia (b)

wave was significantly associated with the occurrence of VT (HR $3.5 ; p=0.019)$ (Table 5).

\section{Discussion}

The existence of $J$ wave on sECG is considered a benign finding observed in approximately $2-10 \%$ of the general population [8]. However, following the findings reported by Haissaguerre et al. [9] and Nam et al. [10] the J wave syndrome has emerged as a significant cause of idiopathic ventricular fibrillation. The concept has now expanded to include other structural heart disease such as acute myocardial infarction, variant angina and even some forms of cardiomyopathy, such as arrhythmogenic right ventricular dysplasia/cardiomyopathy and noncompaction cardiomyopathy. In addition, two important J wave syndromes, the ER and Brugada syndromes (BS) are also considered to be of clinical importance.
J wave on the sECG is believed to originate from the generation of a transmural voltage gradient between the endocardium and epicardium during repolarization or depolarization, due to decreased inward sodium or calcium channel currents, or an increase in outward potassium currents mediated through the $I_{\mathrm{to}}, I_{\mathrm{K}-\mathrm{ATP}}$, and $I_{\mathrm{K} \text {-Ach }}$ channels. These outward current shifts and heterogeneous loss of the action potential (AP) dome result in a marked dispersion of repolarization, which is followed by phase 2 reentry and gives rise to polymorphic VT or VF. Transient ischemia, caused by multivessel coronary spasm or neurological stress, [11] has been suggested as an additional mechanism triggering J wave and VT.

Masato et al. [12], reported a prevalence of J wave of approximately $30 \%$ ( 9 of 30 patients), and of VT/VF in the J wave and non-J wave group of approximately $22 \%$ ( 2 of 9 patients) and 9\% ( 2 of 22 patents), respectively, in patients with TTC. Similarly, in our study, we found 
Table 4 QTc interval and characteristics of the J wave between VT and Non-VT group

\begin{tabular}{|c|c|c|c|c|}
\hline & Total $(n=79)$ & VT group $(n=20)$ & Non-VT group $(n=59)$ & $P$ value \\
\hline Presence of J wave, No (\%) & $30(37)$ & $15(75)$ & $15(25)$ & 0.001 \\
\hline \multicolumn{5}{|l|}{ Slur-Notch, No (\%) } \\
\hline Slur & $4(5)$ & $2(10)$ & $2(3)$ & 0.57 \\
\hline Notch & $26(32)$ & $13(65)$ & $13(21)$ & 0.001 \\
\hline \multicolumn{5}{|l|}{ Localization, No (\%) } \\
\hline Inferior & $21(26)$ & $9(45)$ & $12(20)$ & 0.04 \\
\hline Inferolateral (both)* & $3(3)$ & $2(10)$ & $1(1)$ & 0.15 \\
\hline \multicolumn{5}{|l|}{ Magnitude of J wave, No (\%) } \\
\hline Amplitude $\geq 0.2 \mathrm{mV}$ & $11(13)$ & $9(45)$ & $2(3)$ & 0.001 \\
\hline $\begin{array}{l}\text { J point elevation } \geq 0.2 \mathrm{mV} \& \text { inferolateral } \\
\text { leads location }\end{array}$ & $10(12)$ & $8(40)$ & $2(3)$ & $<0.001$ \\
\hline \multicolumn{5}{|l|}{ J point with ST segment, No (\%) } \\
\hline Ascending & $6(7)$ & $2(10)$ & $4(6)$ & 0.64 \\
\hline Horizontal/descending & $24(30)$ & $13(65)$ & $11(18)$ & $<0.001$ \\
\hline
\end{tabular}

Values are mean \pm SD or number (\%); VT ventricular tachycardia; Inferolateral* J wave in at least two inferior leads and in two lateral leads; Inferolateral location ${ }^{\dagger} \mathrm{J}$ wave in at least two inferior leads or in two lateral leads, or both

Table 5 Multivariate cox analysis of known risk factors for ventricular tachycardia

\begin{tabular}{llll}
\hline Variables & HR & $\mathbf{9 5 \% ~ C l}$ & P value \\
\hline Univariate regression analysis & & & \\
Age & 1.02 & $0.99 \sim 1.05$ & 0.21 \\
Male sex & 1.84 & $0.73 \sim 4.63$ & 0.19 \\
Hypertension & 0.70 & $0.28 \sim 1.72$ & 0.44 \\
Diabetes Mellitus & 0.89 & $0.33 \sim 2.63$ & 0.89 \\
Smoking & 1.20 & $0.43 \sim 3.33$ & 0.72 \\
QTc duration & 1.01 & $1.00 \sim 1.02$ & 0.002 \\
Peak CK level & 1.00 & $1.00 \sim 1.00$ & 0.26 \\
LVEF & 0.99 & $0.94 \sim 1.06$ & 0.90 \\
ST-segment elevation & 1.06 & $0.44 \sim 2.56$ & 0.90 \\
Presence of J wave & 5.16 & $1.87 \sim 14.27$ & 0.002 \\
Multivariate regression analysis & & & \\
QTc duration & 1.01 & $1.00 \sim 1.01$ & 0.06 \\
Presence of J wave & 3.53 & $1.23 \sim 10.17$ & 0.019 \\
\hline
\end{tabular}

$H R$ hazard ratio; $C l$ confidence interval; $L V E F$ left ventricular ejection fraction; $C K$ creatine kinase

J wave prevalence of $37.5 \%$ (30 of 80 patients), and VT prevalence of $53 \%$ (15 of 30 patients) and $8 \%$ (5 of 50 patients) in the J wave and non-J wave group, respectively.

Samuelov-Kinori et al. [13] reported longer QTc intervals in patients with TTC who developed TdP. The mechanisms of QT prolongation in TTC appear to be similar to those of AMI resulting from autonomic dysregulation [14], and the intracardial gradient (apicobasal) of myocardial edema leads to transient inhomogeneity and gives rise to regional dispersion of AP duration [15]. These mechanisms may explain the prolonged QT interval in TTC, and the even longer QT interval seen in the VT and $\mathrm{J}$ wave groups, compared with that in the non-VT and non-J wave groups. Results from univariate and multivariate logistic analyses also showed that a prolonged QT interval appears to be an independent risk factor for VT. Multivariate logistic analyses also indicated that male sex is a risk factor for VT, in agreement with previous studies suggesting that male sex is strongly associated with the ER pattern observed on the ECG [16].

A relationship between the magnitude of the J point elevation and a higher occurrence of VF episodes has been previously reported [9]. Antzelevitch et al. [17] classified the ER pattern into three subtypes to estimate the risk for the development of malignant arrhythmia, according to the ECG leads in which the ER pattern appears. An ER pattern in the lateral leads; inferior or inferolateral leads; and globally, in the inferior, lateral and right precordial leads; is associated with a low, moderate, and high risk, respectively. However, Tikkanen et al. [18] proposed another classification based on the shape of the ST segment after the J wave, with a rapidly ascending ST, and a horizontal or descending ST segment being considered benign and malignant forms. Likewise, we found that the prevalence of J wave with an amplitude $\geq 0.2 \mathrm{mV}$, seen in the inferior leads, or in the inferolateral leads with a J point elevation $\geq 0.2 \mathrm{mV}$ as well as horizontal/ descending ST segments seen after the J point, was significantly higher in the VT group than in the non-VT group.

Our results suggest that the presence of a J wave on the sECG is significantly associated with the occurrence 
of polymorphic VT or TdP during TTC. Therefore, the identification of a J wave on sECG during TTC may help to distinguish patients who are susceptible to developing VT, especially in men with a prolonged QT interval, in whom close monitoring and primary prevention of VT should be considered. Additionally, evidence on the genetic basis for ER is currently limited, but recent reports suggest that mutations in candidate genes such as $K C N J 8$ which encode a pore-forming subunit of the ATP-sensitive potassium channel, as well as CACNA1C, $C A C N B 2$, and $C A C N A 2 D 1$ which encode a L-type calcium channel, and $S C N 5 A$ related to $I_{\mathrm{Na}}$, may be implicated in ER pathophysiology [19-21]. Hence, further studies are needed to clarify the relationship between genetic susceptibility and VT in TTC patients with J wave on sECG.

Our study had several limitations. Firstly, this was a retrospective study, with a small sample size, and limited to a single center. Secondly, since we could not perform stress tests or cardiac magnetic resonance imaging to verify the presence of myocardial scar, which is known to have arrhythmogenic potential, it could not be objectively ruled out. Thirdly, although we monitored cardiac rhythm thoroughly in patients hospitalized in the intensive care unit, this level of monitoring could not be achieved in the general ward. Nevertheless, our results provide valuable evidence of clinical implications of $J$ wave in predicting the development of lethal arrhythmia during TTC. Further multi-center, prospective studies in larger groups of patients, are necessary to confirm the predictive value of $J$ wave.

\section{Conclusion}

In conclusion, our findings suggest that the presence of J wave on the sECG is significantly associated with ventricular arrhythmia during TTC, and appear to indicate that the presence of $\mathrm{J}$ wave is a strong and independent predictor of VT in patients with TTC.

\section{Abbreviations}

TCC: Takotsubo Cardiomyopathy; sECG: Surface Electrocardiography; VT:Ventricular Tachycardia; AMI: Acute Myocardial Infarction; TdP: Torsades de Pointes; ER: Early Repolarization; VF: Ventricular Fibrillation; BS: Brugada Syndromes; AP: Action Potential.

\section{Acknowledgements}

None.

\section{Authors' contributions}

Choi SH, Lee OH and Baek YS involved in writing draft and analyzing data. Kim $\mathrm{DH}$ and Baek YS were involved in writing draft and creating concept of study. KSW, YGS, SSH, PSD, WSI and KJ involved in data review and writing draft. All authors read and approved the final manuscript.

\section{Funding}

This study was supported by Korean Heart Rhythm Society (KHRS 2017-4); and the INHA UNIVERSITY Research Grant; and the INHA UNIVERSITY HOSPITAL
Research Grant; and the National Research Foundation of Korea (NRF) grant funded by the Korea government (MISP) under NRF-2014R1A5A2009392.

\section{Availability of data and materials}

All data generated or analyzed during this study are included in this published article.

\section{Ethics approval and consent to participate}

This study was approved by the Institutional Review Board at Inha University Hospital, which waived the requirement of obtaining written informed consent from patients.

\section{Consent for publication}

Not applicable.

\section{Competing interests}

The authors have declared no competing interests.

Received: 13 October 2019 Accepted: 21 May 2020

Published online: 08 June 2020

\section{References}

1. Hurst RT, Prasad A, Askew JW 3rd, Sengupta PP, Tajik AJ. Takotsubo cardiomyopathy: a unique cardiomyopathy with variable ventricular morphology. JACC Cardiovasc Imaging. 2010;3:641-9.

2. Madias C, Fitzgibbons TP, Alsheikh-Ali AA, Bouchard JL, Kalsmith B, Garlitski $A C$, et al. Acquired long QT syndrome from stress cardiomyopathy is associated with ventricular arrhythmias and torsades de pointes. Heart Rhythm. 2011;8:555-61.

3. Koncz I, Gurabi Z, Patocskai B, Panama BK, Szel T, Hu D, et al. Mechanisms underlying the development of the electrocardiographic and arrhythmic manifestations of early repolarization syndrome. J Mol Cell Cardiol. 2014;68:20-8.

4. Nam GB. Idiopathic ventricular fibrillation, early repolarization and other J wave-related ventricular fibrillation syndromes: from an electrocardiographic enigma to an electrophysiologic dogma. Circ J. 2012;76:2723-31.

5. Prasad A, Lerman A, Rihal CS. Apical ballooning syndrome (tako-tsubo or stress cardiomyopathy): a mimic of acute myocardial infarction. Am Heart J. 2008;155:408-17.

6. Wang J, Tang M, Mao KX, Chu JM, Hua W, Jia YH, et al. Idiopathic ventricular fibrillation with fragmented QRS complex and J wave in resting electrocardiogram. J Geriatr Cardiol. 2012;9:143-7.

7. Ghadri JR, Wittstein IS, Prasad A, et al. International expert consensus document on Takotsubo syndrome (Part I): clinical characteristics, diagnostic criteria, and pathophysiology. Eur Heart J. 2018;39(22):2032-46.

8. Sethi KK, Sethi K, Chutani SK. Early repolarisation and I wave syndromes Indian Heart J. 2014;66:443-52.

9. Haissaguerre M, Derval N, Sacher F, Jesel L, Deisenhofer I, de Roy L, et al. Sudden cardiac arrest associated with early repolarization. N Engl J Med. 2008;358:2016-23.

10. Nam GB, Kim YH, Antzelevitch C. Augmentation of J waves and electrical storms in patients with early repolarization. N Engl J Med. 2008:358:2078-9.

11. Kukla P, Jastrzebski M, Praefort W. J-wave-associated ventricular fibrillation in a patient with a subarachnoid haemorrhage. Europace. 2012;14:1063-4.

12. Shimizu M, Nishizaki M, Yamawake N, Fujii H, Sakurada H, Isobe M, et al. I wave and fragmented QRS formation during the hyperacute phase in takotsubo cardiomyopathy. Circ J. 2014;78:943-9.

13. Samuelov-Kinori L, Kinori M, Kogan Y, Swartzon M, Shalev H, Guy D, et al. Takotsubo cardiomyopathy and QT interval prolongation: who are the patients at risk for torsades de pointes? J Electrocardiol. 2009;42(4):353-7.

14. Ogura R, Hiasa Y, Takahashi T, Yamaguchi K, Fujiwara K, Ohara Y, et al. Specific findings of the standard 12-lead ECG in patients with 'takotsubo' cardiomyopathy: comparison with the findings of acute anterior myocardial infarction. Circ J. 2003:67:687-90.

15. Perazzolo Marra M, Zorzi A, Corbetti F, De Lazzari M, Migliore F, Tona F, et al. Apicobasal gradient of left ventricular myocardial edema underlies 
transient T-wave inversion and QT interval prolongation (wellens'ECG pattern) in tako-tsubo cardiomyopathy. Heart Rhythm. 2013;10:70-7.

16. Junttila MJ, Sager SJ, Tikkanen JT, Anttonen O, Huikuri HV, Myerburg RJ. Clinical significance of variants of J-points and J-waves: early repolarization patterns and risk. Eur Heart J. 2012;33:2639-43.

17. Antzelevitch C, Yan GX. J wave syndromes. Heart Rhythm. 2010;7:549-58.

18. Tikkanen JT, Junttila MJ, Anttonen O, Aro AL, Luttinen S, Kerola T, et al. Early repolarization: electrocardiographic phenotypes associated with favorable long-term outcome. Circulation. 2011;123:2666-73.

19. Haissaguerre M, Chatel S, Sacher F, Weerasooriya R, Probst V, Loussouarn $\mathrm{G}$, et al. Ventricular fibrillation with prominent early repolarization associated with a rare variant of KCNJ8/KATP channel. J Cardiovasc Electrophysiol. 2009;20:93-8.
20. Medeiros-Domingo A, Tan BH, Crotti L, Tester DJ, Eckhardt L, Cuoretti A, et al. Gain-of-function mutation S422L in the KCNJ8-encoded cardiac K(ATP) channel Kir6.1 as a pathogenic substrate for J-wave syndromes. Heart Rhythm. 2010;7:1466-71.

21. Burashnikov E, Pfeiffer R, Barajas-Martinez H, Delpon E, Hu D, Desai M, et al. Mutations in the cardiac L-type calcium channel associated with inherited J-wave syndromes and sudden cardiac death. Heart Rhythm. 2010;7:1872-82.

\section{Publisher's Note}

Springer Nature remains neutral with regard to jurisdictional claims in published maps and institutional affiliations.
Ready to submit your research? Choose BMC and benefit from:

- fast, convenient online submission

- thorough peer review by experienced researchers in your field

- rapid publication on acceptance

- support for research data, including large and complex data types

- gold Open Access which fosters wider collaboration and increased citations

- maximum visibility for your research: over $100 \mathrm{M}$ website views per year

At BMC, research is always in progress.

Learn more biomedcentral.com/submissions 